Relations industrielles

Industrial Relations

\title{
Normes sociales, droit du travail et mondialisation : confrontations et mutations, par Marie-Ange MOREAU, Paris : Dalloz, 2006, 461 p., ISBN 2-247-06950-9.
}

\section{Pierre Verge}

Volume 61, numéro 3, été 2006

URI : https://id.erudit.org/iderudit/014190ar

DOI : https://doi.org/10.7202/014190ar

Aller au sommaire du numéro

Éditeur(s)

Département des relations industrielles de l'Université Laval

ISSN

0034-379X (imprimé)

1703-8138 (numérique)

Découvrir la revue

Citer ce compte rendu

Verge, P. (2006). Compte rendu de [Normes sociales, droit du travail et mondialisation : confrontations et mutations, par Marie-Ange MOREAU, Paris : Dalloz, 2006, 461 p., ISBN 2-247-06950-9.] Relations industrielles / Industrial Relations, 61(3), 537-539. https://doi.org/10.7202/014190ar

Tous droits réservés (C) Département des relations industrielles de l'Université Laval, 2006
Ce document est protégé par la loi sur le droit d'auteur. L’utilisation des services d'Érudit (y compris la reproduction) est assujettie à sa politique d'utilisation que vous pouvez consulter en ligne.

https://apropos.erudit.org/fr/usagers/politique-dutilisation/ 
D'autre part, dans les systèmes de RI de type subordonné les syndicats ne peuvent s'opposer efficacement aux efforts des employeurs de résister à l'accroissement des droits syndicaux ni de faire un lobbying efficace pour réduire les droits syndicaux existant, surtout dans le secteur privé de l'économie comme ce fut le cas en NouvelleÉcosse avec le célèbre Bill Michelin en 1979. L'ouvrage contient également de précieuses informations statistiques qui illustrent bien le propos des auteurs. Ainsi dans les provinces à système de RI subordonné, on constate que la proportion des employés syndiqués provenant du secteur public dans l'ensemble des effectifs syndicaux est plus élevée que dans les provinces à système de RI reconnu. De plus, la proportion de conflits de travail illégaux est également plus élevée que dans l'autre groupe de provinces.

Beyond the National Divide souligne cependant un certain nombre de traits communs aux différents systèmes de RI, notamment : la présence de garanties sur les droits syndicaux fondamentaux et sur le fonctionnement de la négociation collective qui sont supérieures à ce que l'on retrouve dans la législation du travail aux États-Unis et ce, même dans les systèmes de RI de type subordonné; la vigueur de la négociation collective en général au Canada; la difficulté des syndicats à organiser le secteur des services privés; la décentralisation de la négociation collective à peu près partout au Canada, sauf au Québec et en Colombie-Britannique où l'on retrouve des exceptions dans certains secteurs; enfin, une constatation probante: l'importance des facteurs économiques plutôt que politiques dans le profil historique des conflits de travail.

Nous recommandons fortement la lecture de ce livre à toutes les personnes qui s'intéressent à l'analyse du fonctionnement des systèmes de relations industrielles dans les pays industrialisés.

JeAn BoIvin

Université Laval

\section{Normes sociales, droit du travail et mondialisation : confrontations et mutations,}

par Marie-Ange MorEAU, Paris : Dalloz, 2006, 461 p., ISBN 2-247-06950-9.

Parmi tous ces ouvrages contemporains consacrés à l'impact de la mondialisation sur le travail et sa régulation, celui que vient de publier la professeure Marie-Ange Moreau, titulaire de la Chaire de droit social à l'Institut universitaire européen de Florence, occupera une place bien distincte, ne serait-ce que parce qu'il nous livre une réflexion d'ensemble d'un seul et même auteur sur cet ample et mouvant phénomène. Bref, une " analyse générale des liens entre normes sociales, droit du travail et mondialisation » (p. 16) de nature à permettre de visiter les lieux, de différents niveaux, d'élaboration de ces normes et de ce droit, de dégager leur interrelation et de tenter de déceler les orientations de cet ensemble.

Un chapitre préliminaire dégage notamment les caractéristiques les plus significatives de la mondialisation sur le plan social, dont le développement du commerce international et celui des entreprises multinationales. L'activité et les stratégies de ces dernières sont transnationales; les réponses des acteurs sur le plan social doivent être commensurables. Articulation dès lors de règles allant du niveau local au niveau global; un contexte de «pluralisme normatif » s'impose. 
La première partie de l'ouvrage explore, en les confrontant, les différents modes de régulation du travail, les différents mécanismes de protection des travailleurs dans cette économie mondialisée. Concurrence des autorités normatives en premier lieu : entre systèmes nationaux (phénomène du dumping social); entre différents niveaux de régulation : national (remise en cause du rôle de l'État; pressions exercées sur la négociation collective au niveau national); régional (supranationalité régionale de l'Union européenne, comparée aux accords multinationaux dans les Amériques (MERCOSUR, CARICOM et surtout ceux liant les États-Unis et le Canada, dont l'ANACT)); les institutions internationales, au premier chef desquelles l'OIT (examen de sa récente évolution politique, que traduisent en particulier sa Déclaration des principes et droits fondamentaux du travail et son suivi de 1998 et sa politique générale de promotion du «travail décent»). Confrontation, en deuxième lieu, des techniques de régulation; il y va ici de la coexistence des normes de hard law et celles, plus diversifiées et typiquement plus vaporeuses, dites de soft law. Elle conduit à l'examen, relativement détaillé, des techniques d'harmonisation des normes sociales dans l'Union européenne et de la stratégie européenne de l'emploi, de même qu'à une évocation des procédés de coopération interétatique dans le cadre de l'ANACT (ALÉNA), en l'absence alors de «[toute] préoccupation de gouvernance au niveau de l'espace économique [correspondant]» (p. 167). Il faut aussi tenir compte des techniques de régulation développées au sein même de l'entreprise transnationale, soit unilatéralement (codes de conduite), soit avec le concours d'un élément syndical (accordscadres transnationaux d'entreprise) : la construction est ici, horizontalement, à la mesure de l'entreprise transnationale, mais non sans problèmes quant à sa juridicité... Confrontation, enfin, précisément, quant à l'efficacité de ces différents mécanismes de protection des travailleurs (de l'efficacité des clauses sociales dans les traités commerciaux internationaux, c'est-à-dire en définitive, de l'opportunité de sanctions économiques en cas de violations des normes fondamentales du travail (OMC, ANACT, systèmes généralisés de préférence) et de celle des systèmes de traitement classique des plaintes en semblables situations, tant au sein de l'OIT, des institutions du Conseil de l'Europe que dans le cas de l'ANACT). Ainsi, « [...] on assiste à une prolifération des normes de niveaux différents, construites sur des modèles différents, qui montrent la nécessité de dépasser la hiérarchie classique des règles pour construire un niveau transnational » (p. 225).

Quelles tendances dégager du précédent ensemble normatif ? Une seconde partie dégage avec force les «mutations » suivantes. En premier lieu, la reconnaissance d'un "mouvement indiscutable » en faveur de l'affirmation de la centralité des droits sociaux fondamentaux, qu'il s'agisse des constitutions nationales, du renforcement historique de ces droits dans l'Union européenne, ou encore, de la Déclaration de l'OIT de 1998. Cette affirmation doit trouver appui dans la coordination des actions d'affirmation de ces droits sociaux, lesquelles présupposent à leur tour un climat de liberté syndicale (p. 275). Reconnaissance ensuite de l'inadaptation de concepts fondamentaux du droit du travail classique face à l'activité de l'entreprise transnationale (rigidité ainsi de la notion d' «employeur » dans certains droits nationaux), de même que constat de l'insuffisance des règles du droit international privé régissant la mobilité du salarié au sein de cette dernière, a fortiori celles régissant les droits collectifs des salariés mobiles. Retour sur les expressions en soft law de la responsabilité sociale de l'entreprise, pour accentuer les difficultés de leur réception dans les droits nationaux classiques (absence reprochée également à l'OIT 
de tout système de contrôle des codes de conduite privés (p. 324)) et mise en relief de l'originalité du dialogue social européen et de celle de la négociation collective transnationale. Ces dernières normes ont le mérite, souligne-t-on encore une fois, de « montr[er] la possible intégration de l'internationalisation des entreprises dans le droit du travail. [Elles] conduisent à ébranler les modèles traditionnels. Elles sont en développement, en construction » (p. 349). Enfin, à la base, les acteurs et leur évolution : la conjoncture présente des groupements syndicaux et l'adéquation de leur action par rapport à celle des entreprises transnationales; les réticences des organisations d'employeurs européennes à favoriser la négociation collective au plan européen; la problématique de la représentativité des ONG et de la légitimité de leur action; l'émergence d'alliances syndicales transnationales, y compris le concours d'organismes de défense des droits de la personnes, par exemple à l'occasion de la présentation de communications du public selon l'ANACT; le tripartisme dans certains pays et au sein de l'Union européenne... Une place spéciale, en fin d'analyse, est réservée à certaines manifestations jurisprudentielles d'adaptation à la transnationalité du travail, qu'il s'agisse de hautes juridictions européennes, françaises en particulier, ou de certaines espèces de stratégie judiciaire aux États-Unis. En somme, au terme de cette seconde partie, " [l'] apparition de normes transnationales dans le domaine des relations du travail montre que le droit du travail - au sens large du terme - a la capacité de se reconfigurer en fonction des figures de l'employeur : à l'employeur transnational, normes du travail transnationales » (p. 408). À la fois, peut-on dire à cet égard, une réalité déjà émergente, mais une aspiration à plus.... Recherche en définitive de cohérence et de concordance dans, à la fois, ces « exigences de dépassement des cadres nationaux » et cette nécessaire "diversification des modes de régulation », pour assurer la protection des travailleurs face à la mondialisation (p. 412).

Normes de différents niveaux, pluralisme juridique : la synthèse est considérable et innovatrice avions-nous constaté. L'auteure entendait bien d'ailleurs présenter une "analyse générale des liens entre normes sociales, droit du travail et mondialisation... » (p. 16), s'engager dans une « recherche du mouvement des mécanismes de régulation sociale et de l'identification des voies qui s'ouvrent aux différents acteurs »(p.14). À ces fins, elle a su exploiter un ensemble doctrinal fort riche et rendre compte des travaux les plus récents, voire de recherches en cours dans plusieurs des domaines abordés. Faisant elle-même partie des membres fondateurs du Centre de recherche interuniversitaire sur la mondialisation et le travail (CRIMT) au Canada, les travaux de ce groupe de chercheurs sont ainsi particulièrement mis à contribution. Ces repères se rattachent à toutes les grandes régions du globe. Une mise en rapport accentuée de la littérature européenne et de celle de provenance nord-américaine constitue une autre caractéristique innovatrice et stimulante de l'ouvrage.

En particulier et d'un point de vue nord-américain, les institutions et les pratiques régionales européennes peuvent certes avoir valeur d'exemplarité; des différences contextuelles majeures les éloignent toutefois de la réalité nord-américaine, ce que l'auteure ellemême met d'ailleurs bien en relief. Cette dernière réalité pourrait ultérieurement conduire à accentuer de façon complémentaire l'analyse comparée des techniques d'adaptation des droits nationaux régissant le travail à la réalité de l'activité des entreprises transnationales; le droit de portée horizontale correspondant à ces dernières semble, en effet, destiné à conserver en moyenne portée ses caractéristiques de soft law.

Pierre Verge Université Laval 